\title{
APLICACIÓN DEL PROGRAMA DE HABILIDADES COMUNICATIVAS PARA LA RESOLUCIÓN PACÍFICA DE CONFLICTOS EN EL AULA DEL QUINTO GRADO DE PRIMARIA DE LA I.E. MARÍA UGARTECHE DE MAC LEAN DE TACNA DEL AÑO 2011
}

\author{
APPLICATION COMMUNICATION SKILLS PROGRAM FOR \\ PEACEFUL CONFLICT RESOLUTION IN THE CLASSROOM OF \\ FIFTH GRADE OF EI MARY MAC UGARTECHE OF LEAN OF \\ THE YEAR 2011 TACNA
}

Yeni Gladys Mayta Mamani ${ }^{1}$

\begin{abstract}
RESUMEN
Objetivo: Determinar si la aplicación del "Programa de habilidades comunicativas" mejora la resolución pacífica de conflictos en el aula de las niñas del quinto grado de primaria de la I.E. "María Ugarteche de Mac Lean" de Tacna.

Método: Diseño cuasi-experimental, se aplicó el "Programa de Habilidades Comunicativas" al grupo experimental, así como, un cuestionario de valoración del programa; y, cuestionarios de pre-test y post-test a los grupos de control y experimental; formaron parte del estudio 60 niñas del quinto grado de educación primaria. Resultados: Mejora considerable en cuanto a la "Escucha Activa", mejora significativamente de la "Asertividad". Las "Técnicas para afrontar conflictos" tienen un alto incremento, la resolución del conflicto a través de un enfoque colaborativo.

Conclusión: El desarrollo de las habilidades comunicativas, para la resolución pacífica de conflictos en el aula, mejora en el grupo experimental de las niñas del quinto grado de primaria de la I.E. "María Ugarteche de Mac Lean" y es superior al grupo de control, después de aplicar el programa. Es eficaz y estadísticamente significativa.
\end{abstract}

PALABRAS CLAVES: conflicto, habilidades comunicativas, asertividad.

\begin{abstract}
Objetive: Determine whether the application of "Communication Skills Program" improves the peaceful resolution of conflicts in the classroom of girls in the fifth grade of the I.E. "Maria Ugarteche de Mac Lean" school of Tacna.

Method: Using quasi-experimental design, "Communication Skills Program" was applied to the experimental group and a program evaluation questionnaire as well; and pre-test questionnaires and post-test to the experimental and control groups; 60 girls in the fifth grade of primary education were part of the research.

Result: Considerable improvement as to the "Active Listening", significantly improves of "assertiveness". The "Techniques for confront conflicts" have high increment, resolving the conflict through a collaborative approach.

Conclusion: The development of communication skills for the peaceful resolution of conflicts in the classroom, improvement in the experimental group of girls in the fifth grade of primary of I.E. "Mary Mac Lean Ugarteche" and is superior to the control group, after applying the program. It is effective and statistically significant.
\end{abstract}

KEY WORDS: conflicts, communication skills, assertiveness.

\footnotetext{
Tesis de postgrado para optar el Grado de Mg. en psicopedagogía. Escuela de Postgrado - Universidad Privada de Tacna.

E-mail:yenimayta@hotmail.com
} 


\section{INTRODUCCIÓN}

En todas las culturas y a lo largo del tiempo han existido los conflictos entre los seres humanos, estos forman y formarán parte de nosotros, están presentes en todas las relaciones humanas. Si abordamos los conflictos correctamente, no a través de la violencia sino de la escucha activa, el diálogo, el respeto mutuo, la negociación y el autocontrol, es decir, utilizando todas aquellas acciones encaminadas a luchar contra el conflicto y no contra la persona, lograremos un resultado positivo de los mismos, un crecimiento y enriquecimiento personal. "Sería imposible pensar en una comunidad grande o pequeña que no tuviera conflictos y en la que nunca entraran en desacuerdo las personas".

En el ámbito educativo los conflictos se pueden abordar a través de programas de intervención debidamente estructurados. El plan de acción tutorial de aula permite, hacer efectiva la labor de acompañamiento a los estudiantes, organizar lo que va a realizarse de manera concreta considerando sus características, necesidades e intereses.

La I.E. María Ugarteche de Mac Lean tiene como contenido trasversal "La educación en valores para lograr una convivencia armoniosa", es por ello que partimos de la convicción que procurar una buena convivencia no solo es una condición para realizar el trabajo escolar, sino que es un aprendizaje básico en sí mismo al que debe contribuir la educación escolar y que ha de ser resultado de un trabajo sistemático. Así, se puede proponer la elaboración de programas para alcanzar un objetivo significativo, seleccionando actividades ya existentes o creando actividades nuevas. En la elaboración del "Programa de habilidades comunicativas" se desarrollan actividades que ayudan a fortalecer la escucha activa, asertividad, expresión de emociones y estrategias para afrontar conflictos y así contribuir al logro de una convivencia armoniosa, tiene un enfoque de intervención y un enfoque preventivo.

\section{OBJETIVOS DE INVESTIGACIÓN}

\section{Objetivo general}

Determinar si la aplicación del "Programa de habilidades comunicativas" mejora la resolución pacífica de conflictos en el aula de las niñas del quinto grado de primaria de la I.E. "María Ugarteche de Mac Lean de Tacna".

\section{Objetivos específicos}

1. Identificar el estado inicial de las habilidades comunicativas frente a la resolución pacífica de conflictos en el aula de las niñas del quinto grado de primaria de la I.E. "María Ugarteche de Mac Lean" del grupo de control y experimental antes de la aplicación del programa.

2. Aplicar el "Programa de habilidades comunicativas" para la resolución pacífica de conflictos en el aula de las niñas del quinto grado de primaria del grupo experimental de la I. E "María Ugarteche de Mac Lean".

3. Precisar el desarrollo de las habilidades comunicativas del grupo experimental frente al grupo de control respecto a la resolución pacífica de conflictos en el aula de las niñas del quinto grado de primaria de la I.E. "María Ugarteche de Mac Lean", después de la aplicación del programa.

\section{PREGUNTA}

¿La aplicación de un Programa de Habilidades Comunicativas mejorará la resolución pacífica de conflictos en el aula de las niñas del quinto grado de Educación Primaria en la Institución Educativa María Ugarteche de Mac Lean?

\section{HIPÓTESIS DE INVESTIGACIÓN}

\section{Hipótesis general}

La aplicación del "Programa de habilidades comunicativas" al grupo experimental mejora de manera significativa la resolución pacífica de conflictos en el aula de las niñas del quinto grado de primaria de la I. E. "María Ugarteche de Mac Lean de Tacna”.

\section{Hipótesis específicas}

H1. El estado inicial de las habilidades comunicativas frente a la resolución pacífica de conflictos en el aula de las niñas del quinto grado de primaria de la I.E. "María Ugarteche de Mac Lean" del grupo control y experimental son semejantes antes de la aplicación del programa.

H2. La aplicación del "Programa de habilidades comunicativas" mejora la resolución pacífica de conflictos en el aula de las niñas del quinto grado de primaria del grupo experimental de la I. E "María Ugarteche de Mac Lean".

H3. El desarrollo de las habilidades comunicativas del grupo experimental para la resolución pacífica de conflictos en el aula de las niñas del quinto grado de 
primaria de la I. E "María Ugarteche de Mac Lean" es superior al grupo de control, después de la aplicación del programa.

\section{JUSTIFICACIÓN}

El Diseño Curricular Nacional (DCN) de Educación Básica Regular (EBR) propone temas transversales que responden a problemas de la realidad educativa, uno de ellos, la educación para la convivencia, la paz y la ciudadanía y como un eje curricular el de "Aprender a vivir juntos”. Para lograrlo, la Educación para la Paz busca modificar las actitudes, creencias y comportamientos desde el quehacer cotidiano hasta las negociaciones entre los países a fin que la respuesta a cualquier conflicto sea pacífica, creativa y razonada. Siendo una necesidad educativa la convivencia escolar armoniosa, donde exista el respeto mutuo, la aceptación de normas comunes, aceptación de otras opiniones, estilos de vida y resolución de conflictos por medios pacíficos; además, es una forma de orientar el proceso de desarrollo de los estudiantes para su formación integral como propone la Tutoría y Orientación Educativa (TOE), no solo el aspecto cognitivo del estudiante sino de contribuir al aspecto afectivo y social.

La tutoría en el nivel primario debe crear un clima favorable en el aula, que contribuya a desarrollar el valor del respeto, incentivar la comunicación asertiva para la resolución de problemas y promover el buen trato y la defensa de los derechos. Todos hemos comprobado que, cuando aparecen problemas importantes en la vida, determinadas habilidades son las que nos ayudan a solucionarlos, es nuestra madurez emocional la que nos suele guiar cuando nos tenemos que enfrentar a situaciones difíciles o tareas muy importantes, es por ello que se propone "El Programa de Habilidades Comunicativas" para la resolución pacífica de conflictos en el aula, programa que pretende desarrollar y fortalecer en las niñas del quinto grado de educación primaria el aprender a ser mejores personas, aprender a convivir y a actuar resolviendo pacíficamente conflictos que surgen de las interrelaciones con sus pares .

\section{MATERIALES Y MÉTODOS}

El tipo de investigación corresponde a una investigación Aplicada, tendientes a establecer la relación causa - efecto aplicando a un grupo de niñas del quinto grado primaria.

Corresponde a un diseño Transversal de tipo Cuasiexperimental, con un diseño aplicado a un grupo de control y a un grupo experimental con pre test y post test para ambos.

\section{Técnicas e instrumentos}

\begin{tabular}{|c|c|}
\hline TÉCNICAS & INSTRUMENTOS \\
\hline $\begin{array}{l}\text { Análisis } \\
\text { bibliográfico. }\end{array}$ & $\begin{array}{l}\text {-Fichas de resumen. } \\
\text {-Registros documentarios. }\end{array}$ \\
\hline $\begin{array}{l}\text { Programa de } \\
\text { Habilidades } \\
\text { Comunicativas. }\end{array}$ & $\begin{array}{l}\text {-Registro de conductas. } \\
\text {-Fichas de aplicación. } \\
\text {-Diferencial semántico } \\
\text {-Test de valoración del } \\
\text { programa }\end{array}$ \\
\hline Encuesta. & $\begin{array}{l}\text {-Cuestionario. } 16 \text { ítems en } \\
\text { sentido directo y } 4 \text { en } \\
\text { sentido contrario }(8,10,12 \text { y } \\
\text { 16). }\end{array}$ \\
\hline Observación & $\begin{array}{l}\text { - Lista de cotejo. } \\
\text { - Ficha de observación. }\end{array}$ \\
\hline
\end{tabular}

\section{Procesamiento y análisis de la información}

Los resultados de la aplicación de los instrumentos se presentan en cuadros y gráficos estadísticos e interpretación de los mismos. Se presentan mediante cantidades (n) y en porcentajes (\%).

Para la contrastación de hipótesis se utilizó el coeficiente de correlación de Sperman y estadístico Chi cuadrado. Se utilizó el valor p significativo menor a 0,05 .

\section{RESULTADOS}

RESULTADOS COMPARADOS DE PRE Y POST TEST PARA GRUPO DE CONTROL Y EXPERIMENTAL.

CUADRO $\mathrm{N}^{\circ} 01$

Resultados comparados de la variable Resolución pacífica de conflictos para la dimensión "Escucha activa".

\begin{tabular}{|l|c|c|c|c|}
\hline \multirow{2}{*}{} & \multicolumn{2}{|c|}{ Grupo control } & \multicolumn{2}{c|}{ Grupo experimental } \\
\cline { 2 - 5 } & $\begin{array}{c}\text { Pre- } \\
\text { test }\end{array}$ & Post-test & Pre-test & Post-test \\
\hline Siempre & $34,7 \%$ & $39,3 \%$ & 27,3 & 66,7 \\
\hline A veces & $53,3 \%$ & $54,0 \%$ & 63,3 & 33,3 \\
\hline Pocas veces & $12,0 \%$ & $6,7 \%$ & 9,3 & 0 \\
\hline \multicolumn{1}{|c|}{ Total } & 100 & 100 & 100 & 100 \\
\hline
\end{tabular}

Fuente: Encuesta aplicada a estudiantes. Elaboración: Propia. 


\section{GRÁFICO $\mathbf{N}^{\circ} 01$}

Resultados comparados de la variable Resolución pacífica de conflictos para la dimensión "Escucha activa".

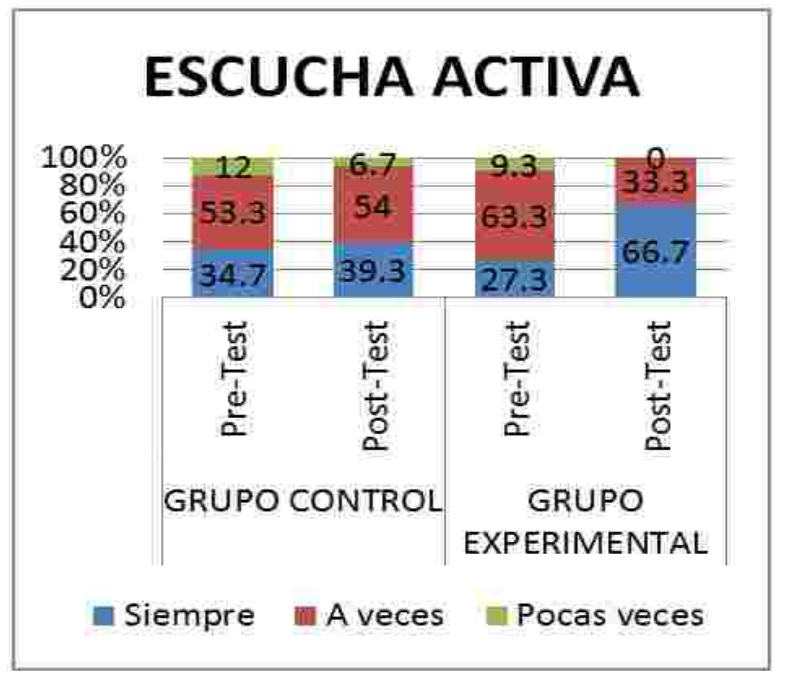

Fuente: Cuadro $\mathrm{N}^{\mathrm{o}} 01$ Elaboración: Propia.

En el cuadro y gráfico $\mathrm{N}^{\circ} 01$, referente a la dimensión "Escucha activa" para el grupo de control, comparando el pre-test y el post-test no observamos una diferencia significativa. Manifiestan un bajo incremento de 34,7\% a $39,3 \%$ en el nivel siempre de la escala.

Para el grupo experimental, comparando pre-test y posttest se observa una diferencia significativa incrementándose de $27,3 \%$ a $66,7 \%$ en el nivel siempre de la escala. Esto muestra que es posible mejorar la habilidad "Escucha activa" después de participar en el programa de habilidades comunicativas.

CUADRO $\mathrm{N}^{\circ} 02$

Resultados comparados de la variable Resolución pacífica de conflictos para la dimensión "Expresión de sentimientos".

\begin{tabular}{|c|c|c|c|c|}
\hline \multirow{2}{*}{} & \multicolumn{2}{|c|}{ Grupo control } & \multicolumn{2}{c|}{ Grupo experimental } \\
\cline { 2 - 5 } & $\begin{array}{c}\text { Pre- } \\
\text { test }\end{array}$ & Post-test & Pre-test & Post-test \\
\hline Siempre & 48,7 & 50,7 & 45,3 & 74 \\
\hline A veces & 37,3 & 39,3 & 40 & 25,3 \\
\hline $\begin{array}{l}\text { Pocas } \\
\text { veces }\end{array}$ & 14 & 10 & 14,7 & 0,7 \\
\hline Total & 100 & 100 & 100 & 100 \\
\hline
\end{tabular}

Fuente: Encuesta aplicada a estudiantes. Elaboración: Propia.
GRÁFICO No 02

Resultados comparados de la variable Resolución pacífica de conflictos para la dimensión "Expresión de sentimientos".

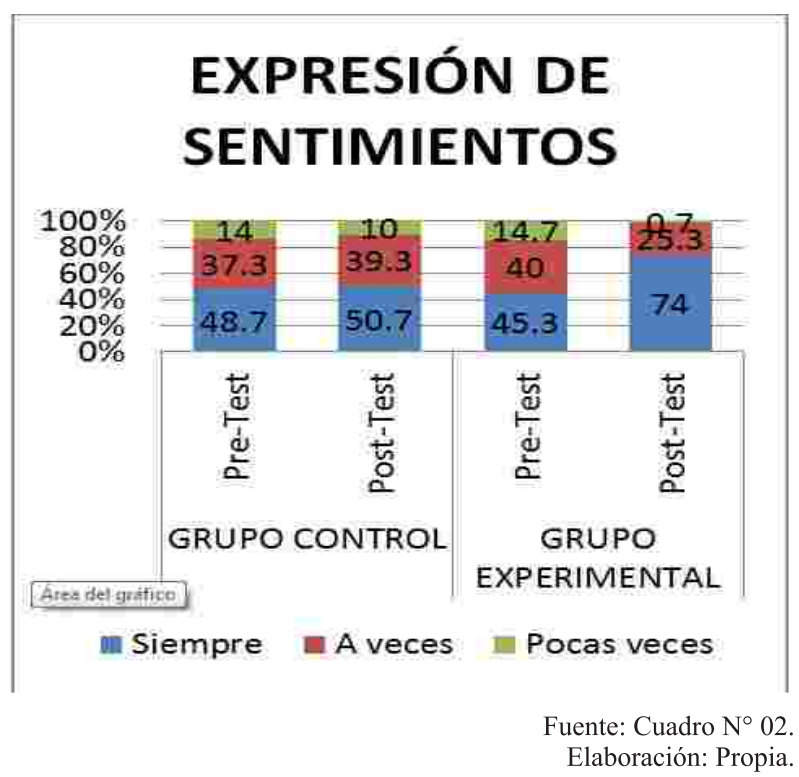

En el cuadro y gráfico $\mathrm{N}^{\circ} 02$, referente a la dimensión "Expresión de sentimientos" para el grupo de control, comparando el pre-test y el post-test no observamos una diferencia significativa. Manifiestan un bajo incremento de $48,7 \%$ a $50,7 \%$ en el nivel siempre de la escala. Para el grupo experimental, comparando el pre-test y el post-test se observa una diferencia significativa incrementándose de $45,3 \%$ a $74 \%$ en el nivel siempre de la escala. Se muestra que es posible mejorar la habilidad "Expresión de sentimientos" después de participar en el programa de habilidades comunicativas para la resolución pacífica de conflictos en el aula.

CUADRO $\mathrm{N}^{\circ} 03$

Resultados comparados de la variable Resolución pacífica de conflictos para la dimensión

"Asertividad".

\begin{tabular}{|l|r|r|r|r|}
\hline \multirow{2}{*}{} & \multicolumn{2}{|c|}{ Grupo control } & \multicolumn{2}{c|}{ Grupo experimental } \\
\cline { 2 - 5 } & $\begin{array}{c}\text { Pre- } \\
\text { test }\end{array}$ & Post-test & Pre-test & Post-test \\
\hline Siempre & 33,3 & 32,7 & 32,7 & 64,7 \\
\hline A veces & 43,3 & 50,7 & 52,7 & 33,3 \\
\hline $\begin{array}{l}\text { Pocas } \\
\text { veces }\end{array}$ & 23,3 & 16,6 & 14,6 & 2 \\
\hline \multicolumn{1}{|c|}{ Total } & 100 & 100 & 100 & 100 \\
\hline
\end{tabular}

Fuente: Encuesta aplicada a estudiantes. Elaboración: Propia. 
GRÁFICO N $^{\circ} 03$

Resultados comparados de la variable Resolución pacífica de conflictos para la dimensión

"Asertividad".

\section{ASERTIVIDAD}

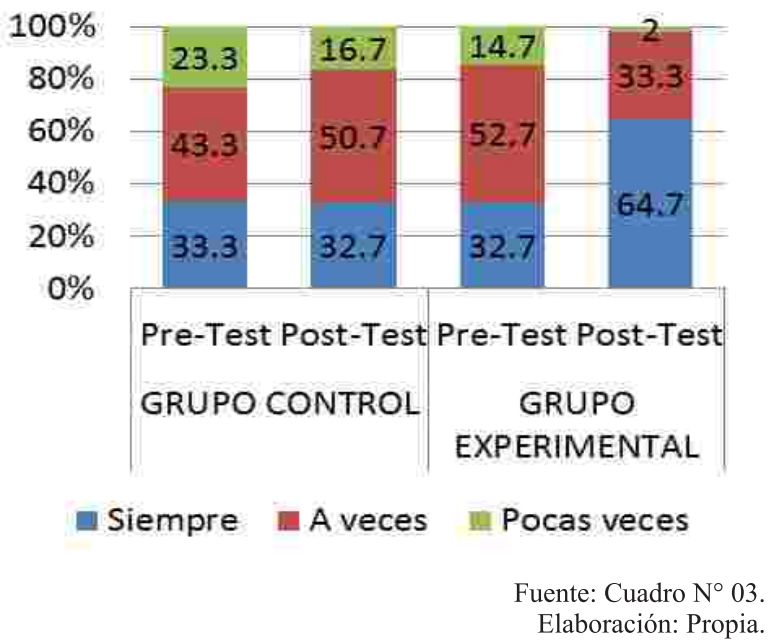

En el cuadro y gráfico $\mathrm{N}^{\circ} 03$, referente a la dimensión "Asertividad" para el grupo de control, comparando el pre-test y el post-test no observamos una diferencia significativa.

Para el grupo experimental, comparando el pre-test y el post-test se observa una diferencia significativa incrementándose de $32,7 \%$ hasta $64,7 \%$ en el nivel siempre de la escala. Se muestra que es posible mejorar la habilidad "Asertividad" después de participar en el programa.

\section{CUADRO $\mathrm{N}^{\circ} 04$}

Resultados comparados de la variable Resolución pacífica de conflictos para la dimensión "Técnicas para afrontar conflictos”.

\begin{tabular}{|l|r|r|r|r|}
\hline & \multicolumn{2}{|c|}{ Grupo control } & \multicolumn{2}{c|}{ Grupo experimental } \\
\cline { 2 - 5 } & Pre-tes! & Post-test & Pre-tesi & \multicolumn{1}{c|}{ Post-test } \\
\hline Siempre & 19,3 & 22 & 13,3 & 71,3 \\
\hline A veces & 50,7 & 56 & 62,7 & 28,7 \\
\hline Pocas veces & 30 & 22 & 24 & 0 \\
\hline Total & 100 & 100 & 100 & 100 \\
\hline
\end{tabular}

Fuente: Encuesta aplicada a estudiantes. Elaboración: Propia.
GRÁFICO No 04

Resultados comparados de la variable Resolución pacífica de conflictos para la dimensión "Técnicas para afrontar conflictos".

\section{TÉCNICAS PARA} AFRONTAR CONFLICTOS

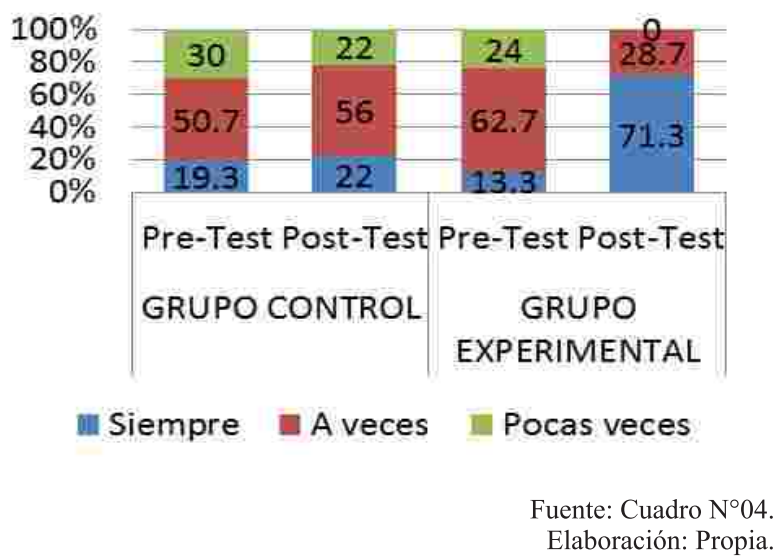

En el cuadro y gráfico $\mathrm{N}^{\circ} 04$, referente a la dimensión "Técnicas para afrontar conflictos" para el grupo de control, comparando el pre-test y el post-test no observamos una diferencia significativa. Manifiestan un incremento de $19,3 \%$ a $22 \%$ en el nivel siempre de la escala.

Para el grupo experimental, comparando el pre-test y el post-test se observa una diferencia significativa incrementándose de $13,3 \%$ hasta $71,3 \%$ en el nivel siempre de la escala. Esto muestra que es posible mejorar la habilidad "Técnicas para afrontar conflictos" después de participar en el "Programa de habilidades comunicativas" para la resolución pacífica de conflictos en el aula.

\section{DISCUSIÓN}

En cuanto a la "Escucha Activa" hay una mejora considerable (Ver Cuadro $\mathrm{N}^{\circ} 31$ ), de 27,3\% subiendo hasta $66,7 \%$, donde se observa que las niñas mostraron un cambio de actitud desde su predisposición a escuchar empáticamente, creando un clima positivo para la comunicación, entendiendo lo que realmente quiere comunicar el emisor, es decir, decodificando el mensaje, respetando a sus compañeras al dialogar con naturalidad., como lo afirma Juan C. Torrego, hay errores que solemos cometer en la comunicación ya sea por el emisor o 
receptor y la escucha activa es una habilidad que minimiza los errores en la comunicación. Respecto a "Expresión de Sentimientos" la mejora es positiva (Ver Cuadro $N^{\circ} 32$ ), de $45,3 \%$ subiendo a $74 \%$; es una habilidad base que necesitamos para desarrollar nuestra capacidad de autocontrol. Creemos que la educación emocional debería ocupar un lugar cada vez más amplio y destacado en nuestro sistema educativo. La alfabetización emocional, como dice Daniel Goleman, contribuye al desarrollo integral de la persona para que pueda ser feliz y contribuya a un ambiente armonioso de convivencia.

La "Asertividad" también mejora significativamente (Ver Cuadro N³3), elevándose de 32,7\% hasta 64,7\%; la conducta asertiva es un aspecto de las habilidades sociales; es el estilo con el que interactuamos. Olga Castanyer afirma que la asertividad es una tercera opción ante un conflicto, no agresiva ni pasiva. La persona asertiva conoce sus propios derechos y los defiende, respeta a los demás, por lo que no piensa ganar en una disputa o conflicto sino que busca de forma positiva los acuerdos.

Las "Técnicas para afrontar conflictos" tienen un alto incremento (Ver Cuadro $\mathrm{N}^{\circ} 34$ ) elevándose de 13,3\% hasta $71,3 \%$; la resolución del conflicto a través de un enfoque colaborativo. Las niñas lograron apropiarse de estrategias como la negociación, en donde ambas partes llegan a un acuerdo que les beneficie, y la mediación donde buscan soluciones las partes involucradas llegando a un acuerdo consensuado. El objetivo principal no es que el profesorado aprenda a resolver los conflictos de las alumnas, sino trabajar con ellos para que aprendan a resolverlos por sí mismos, convirtiendo esa resolución no sólo en un éxito presente, sino en un aprendizaje para otras situaciones.

\section{REFERENCIAS}

1. CASCÓN SORIANO, Paco. (2006). Educar en y para el conflicto. Barcelona: UNESCO.

2. CASTANYER MAYER-SPINES, Olga. (1997). La asertividad expresión de una sana autoestima. Madrid: Desdé de Browser.

3. GOLEMAN, Daniel. (1995). La inteligencia Emocional. Barcelona: Kairós.

4. Ministerio DE EDUCACIÓN. (2009). Aprendiendo a resolver conflictos en las instituciones educativas. Perú: Ditoe.

5. TORREGO, Juan Carlos. (2000). Mediación de conflictos en Instituciones Educativas. Madrid: Narcea.
6. VALLES ARANDIGA, Antonio. (2004). Programa de solución de conflictos interpersonales I. Madrid: EOS. 